\title{
Spin-orbit effects on the Larmor dispersion relation in GaAs quantum wells
}

\author{
Francesc Malet, Enrico Lipparini,* Manuel Barranco, and Martí Pi \\ Departament ECM, Facultat de Física, Universitat de Barcelona. Diagonal 647, 08028 Barcelona, Spain
}

(Received 15 November 2005; published 1 March 2006)

\begin{abstract}
We have studied the relevance of spin-orbit coupling to the dispersion relation of the Larmor resonance observed in inelastic light scattering and electron-spin resonance experiments on GaAs quantum wells. We show that the spin-orbit interaction, here described by a sum of Dresselhaus and Bychkov-Rashba terms, couples Zeeman and spin-density excitations. We have evaluated its contribution to the spin splitting as a function of the magnetic field $B$, and have found that in the small $B$ limit, the spin-orbit interaction does not contribute to the spin splitting, whereas at high magnetic fields it yields a $B$ independent contribution to the spin splitting given by $2\left(\lambda_{R}^{2}-\lambda_{D}^{2}\right)$, with $\lambda_{R, D}$ being the intensity of the Bychkov-Rashba and Dresselhaus spin-orbit terms.
\end{abstract}

DOI: 10.1103/PhysRevB.73.125302

PACS number(s): 73.21.Fg, 71.70.Ej

\section{INTRODUCTION}

The study of spin-orbit (SO) effects in semiconductor nanostructures has been the object of many experimental and theoretical investigations in the last few years, see, e.g., Refs. 1-14 and references therein. It links the spin and the charge dynamics, hence opening the possibility of spin control by means of electric fields. ${ }^{15,16}$

It has been recently shown ${ }^{17}$ that the SO interaction affects the optical properties of GaAs quantum wells by inducing a coupling between charge density and spin density excitations in the long wavelength limit. We extend here this study to the influence on the Larmor resonance of the combined effect of both Dresselhaus ${ }^{18}$ and Bychkov-Rashba ${ }^{19,20}$ SO interactions, and use our results to discuss some features of the spin modes disclosed by inelastic light scattering ${ }^{21,22}$ and electron-spin resonance experiments. ${ }^{23,24}$

Our approach is based on the solution of the equation of motion up to second order in the SO intensity parameters. ${ }^{17}$ This method has been also used to derive the Kohn theorem, ${ }^{25}$ and goes as follows. We write the Schrödinger equation for a $N$-particle system as $H|n\rangle=E_{n}|n\rangle$, with $|0\rangle$ and $E_{0}$ being the ground state (gs) and gs energy, respectively. If one can find an operator $O_{n}^{+}$such that $|n\rangle=O_{n}^{+}|0\rangle, O_{n}|0\rangle=0$, it is possible to cast the Schrödinger equation into an operator equation-the equation of motion- $\left[H, O_{n}^{+}\right]=\omega_{n} O_{n}^{+}$, where $\omega_{n}=E_{n}-E_{0}$ is the excitation energy of the state $|n\rangle$. The solutions of this equation are used to find the excitation energies of the system as well as its excited states in terms of their creation operators.

This work is organized as follows. In Sec. II we apply the equation of motion method to the Larmor mode in the presence of a SO coupling. The results are used in Sec. III to discuss the spin modes in quantum wells, and are compared with the experimental results of Refs. 21 and 24 .

\section{THE EQUATION OF MOTION APPROACH AND THE LARMOR MODE}

The operators describing the SO Rashba and Dresselhaus interactions are respectively given by

$$
H_{R}=\frac{\lambda_{R}}{\hbar} \sum_{j=1}^{N}\left[P_{y} \sigma_{x}-P_{x} \sigma_{y}\right]_{j}
$$

and

$$
H_{D}=\frac{\lambda_{D}}{\hbar} \sum_{j=1}^{N}\left[P_{x} \sigma_{x}-P_{y} \sigma_{y}\right]_{j},
$$

where the $\sigma$ 's are the Pauli matrices and $\mathbf{P}=-i \hbar \nabla+(e / c) \mathbf{A}$ represents the canonical momentum in terms of the vector potential $\mathbf{A}$ which in the following we write in the Landau gauge, $\mathbf{A}=B(0, x, 0)$, with $\mathbf{B}=\nabla \times \mathbf{A}=B \hat{\mathbf{z}}$.

In the effective mass, dielectric constant approximation, the quantum well Hamiltonian $H$ can be quite generally written as $H=H_{K S}+V_{\text {res }}$, where $H_{K S}$ is the Kohn-Sham (KS) onebody Hamiltonian consisting of the kinetic, Rashba, Dresselhaus, exchange-correlation KS potential and Zeeman terms, and $V_{\text {res }}$ is the residual Coulomb interaction. The $K S$ Hamiltonian reads

$$
\begin{aligned}
H_{K S}= & \sum_{j=1}^{N}\left[\frac{P^{+} P^{-}+P^{-} P^{+}}{4 m}+\frac{\lambda_{R}}{2 i \hbar}\left(P^{+} \sigma_{-}-P^{-} \sigma_{+}\right)\right. \\
& \left.+\frac{\lambda_{D}}{2 \hbar}\left(P^{+} \sigma_{+}+P^{-} \sigma_{-}\right)+W_{x c}(n, \xi, \mathcal{V}) \sigma_{z}+\frac{1}{2} g^{*} \mu_{B} B \sigma_{z}\right]_{j},
\end{aligned}
$$

where $m=m^{*} m_{e}$ is the effective electron mass in units of the bare electron mass $m_{e}, P^{ \pm}=P_{x} \pm i P_{y}$, and $\sigma_{ \pm}=\sigma_{x} \pm i \sigma_{y}$. Although other approaches may be also considered, we have considered the exchange-correlation potential $W_{x c}(n, \xi, \mathcal{V})$ in the local-spin current density approximation (LSCDA). ${ }^{26,27} \mathrm{It}$ depends on the density $n$, magnetization $\xi=n^{\uparrow}-n^{\downarrow}$, and local vorticity $\mathcal{V}$, and is evaluated from the exchange-correlation energy per electron $\mathcal{E}_{x c}$ as $W_{x c}=\partial\left(n \mathcal{E}_{x c}\right) / \partial \xi$. The last term in Eq. (3) is the Zeeman energy, where $\mu_{B}=\hbar e /\left(2 m_{e} c\right)$ is the Bohr magneton, and $g^{*}$ is the effective gyromagnetic factor. For bulk GaAs, $g^{*}=-0.44, m^{*}=0.067$, and the dielectric constant is $\epsilon=12.4$. To simplify the expressions, in the following we shall use effective atomic units $\hbar=e^{2} / \epsilon=m=1$. 
In the following, the residual Coulomb interaction will be treated in the adiabatic time-dependent LSCDA (TDLSCDA). ${ }^{27}$ We are going to see that, in the absence of SO coupling, not only the exact Hamiltonian, but also the one in which the residual interaction is treated in the TDLSCDA fulfill the equation

$$
\left[H, S_{\mp}\right]= \pm \omega_{L} S_{\mp},
$$

where $S_{\mp}=1 / 2 \Sigma_{j} \sigma_{\mp}^{j}$ and $\omega_{L}=\left|g^{*} \mu_{B} B\right|$. Thus, if $|0\rangle$ is the gs of the system, the states $S_{\mp}|0\rangle$ are eigenstates of $H$ with excitation energies $\pm \omega_{L}$. Note that a negative $g^{*}$ implies that the spin-up states are lower in energy than the spin-down ones, and that the actual physical solution of Eq. (4) is that corresponding to the $S_{-}$operator. This is the physical contents of the Larmor theorem. Note also that in the absence of spin-orbit coupling, $\left[H, \Sigma_{j}^{N} P_{j}^{+}\right]=\omega_{c} \Sigma_{j}^{N} P_{j}^{+}$, where $\omega_{c}$ $=e B /(m c)$ is the cyclotron frequency. This is the Kohn theorem, which also holds in the adiabatic time-dependent local spin density approximation (TDLSDA) and in the TDLSCDA, and can be generalized to the case of quantum wires and dots parabolically confined.

Since

$$
\left[H, S_{-}\right]=\omega_{L} S_{-}+4 \sum_{j=1}^{N}\left[\lambda_{D} P^{+} \sigma_{z}+i \lambda_{R} P^{-} \sigma_{z}\right]_{j},
$$

the spin-orbit terms in Eq. (3) mix the transverse spin excitations induced by the operator $S_{-}$with the spin-density excitations induced by $\sum_{j=1}^{N} P_{j}^{ \pm} \sigma_{z}^{j}$, and thus Larmor's theorem is not fulfilled. In the following, we use the equation of motion approach to find the eigenvalues and eigenstates of the KS Hamiltonian $H_{K S}$ Eq. (3) which arise from the SO mixing, and will evaluate the spin wave dispersion relation $\omega(q)$ by taking into account the effect of the residual interaction. This is done by first solving the equation of motion

$$
\left[H_{K S}, O^{+}\right]=\omega O^{+}
$$

and then calculate the transverse response $\chi_{t}(q, \omega)$ per unit surface $\mathcal{A}$ in the TDLSCDA

$$
\chi_{t}(q, \omega)=\frac{\chi_{t}^{K S}(q, \omega)}{1-2 F_{x c} \chi_{t}^{K S}(q, \omega)}
$$

where $F_{x c}=W_{x c} / \xi$, and $\chi_{t}^{K S}(q, \omega)$ is the KS transverse response per unit surface. ${ }^{27}$ The poles of $\chi_{t}(q, \omega)$ yield $\omega(q)$. The transverse spin response without inclusion of spin-orbit coupling has been studied in the past in the random-phase approximation $^{28}$ (RPA) and time-dependent Hartree-Fock ${ }^{29}$ approximations.

Up to second order in $\lambda_{R, D}$, Eq. (6) is straighforwardly solved by the operator $O^{+}=\sum_{j=1}^{N}\left[a \sigma_{-}+b P^{+} \sigma_{z}+c P^{-} \sigma_{z}\right] j$. To do so, one has to use the commutators $\left[\sigma_{+}, \sigma_{-}\right]=4 \sigma_{z}$ and $\left[P^{-}, P^{+}\right]=2 \omega_{c}$. This yields a homogeneous system of linear equations for the coefficients $a, b$, and $c$ from which the energies $\omega$ are obtained by solving the secular equation valid up to $\lambda_{R, D}^{2}$ order

$$
\left(\omega-\widetilde{\omega}_{L}\right)\left(\omega^{2}-\omega_{c}^{2}\right)-2 \omega_{c}\left(\lambda_{D}^{2}+\lambda_{R}^{2}\right) \omega-2 \omega_{c}^{2}\left(\lambda_{D}^{2}-\lambda_{R}^{2}\right)=0,
$$

where $\widetilde{\omega}_{L} \equiv\left|g^{*} \mu_{B} B+2 W_{x c}\right|$. This cubic equation can be exactly solved, yielding the excitation energies (only positive solutions are physical). For each of them, the homogenous linear system, supplemented with the normalization condition $\left\langle 0\left|\left[\left(O^{+}\right)^{\dagger}, O^{+}\right]\right| 0\right\rangle=1$, determines the coefficients $a, b$, and $c$. We have found it more convenient to discuss the solutions of the above equation in the limits of small and large magnetic fields, which are more transparent and easier to compare with available experimental data. In the small $B$ limit $\left(\widetilde{\omega}_{L}, \omega_{c} \ll \lambda_{R}, \lambda_{D}\right)$ we obtain a unique solution

$$
\omega=\sqrt{2 \omega_{c}\left(\lambda_{R}^{2}+\lambda_{D}^{2}\right)} .
$$

In the large $B$ limit we obtain

$$
\omega\left(S_{-}\right)=\widetilde{\omega}_{L}+2 \lambda_{R}^{2} \frac{\omega_{c}}{\omega_{c}+\widetilde{\omega}_{L}}-2 \lambda_{D}^{2} \frac{\omega_{c}}{\omega_{c}-\widetilde{\omega}_{L}},
$$

which is mainly excited by the operator $S_{-}$and

$$
\begin{gathered}
\omega\left(P^{+} \sigma_{z}\right)=\omega_{c}+2 \lambda_{D}^{2} \frac{\omega_{c}}{\omega_{c}-\widetilde{\omega}_{L}} \\
\omega\left(P^{-} \sigma_{z}\right)=-\left(\omega_{c}+2 \lambda_{R}^{2} \frac{\omega_{c}}{\omega_{c}+\widetilde{\omega}_{L}}\right),
\end{gathered}
$$

which are mainly excited by the operators $\sum_{j=1}^{N}\left[P^{+} \sigma_{z}\right]_{j}$ and $\sum_{j=1}^{N}\left[P^{-} \sigma_{z}\right]_{j}$. By mainly we mean that the coefficient of the corresponding operator entering the definition is $O\left(\lambda_{R, D}^{0}\right)$, whereas all the others are $O\left(\lambda_{R, D}^{2}\right)$. Note that if $\lambda_{R, D}=0$, the two physical modes in the preceding equations are uncoupled.

Equation (9) shows that, at $B \sim 0$, to order $\lambda_{R, D}^{2}$ there is no spin splitting due to the SO coupling. Indeed, when $B \rightarrow 0$ not only $\omega_{L}$ and $\omega_{c}$ vary linearly with $B$, but also $W_{x c}$ does, implying that the solution of Eq. (8) goes to zero in this limit. Earlier electron-spin resonance measurements on GaAs quantum wells ${ }^{23}$ seemed to indicate that a finite spin splitting was present in the $B=0$ limit. However, subsequent experiments carried out by the same group ${ }^{24}$ covering a broader $B$ range point out that the spin splitting of a Landau level is an exact quadratic function of $B$, and that its extrapolation to $B=0$ leads to a vanishing spin splitting. Our result, which is not changed by the effect of the residual interaction, is thus in full agreement with the experimental findings of Dobers et al. $^{24}$

We have checked that, at low $B$ fields, the dominant component of $\mathrm{O}^{+}$corresponding to the energy Eq. (9) is the spinflip operator $\sum_{j=1}^{N}\left[\sigma_{-}\right]_{j}$. In this limit, the Dresselhaus and Rashba SO interactions act "in phase," whereas at high $B$ they partially compensate each other [compare the energy given in Eq. (9) with these in Eqs. (10) and (11)]. This arises from the structure of the secular Eq. (8), where in the low $B$ limit, the second term dominates over the third one, whereas in the high $B$ limit both terms are equally important, yielding the solutions shown in Eqs. (10) and (11). We have not been able to find a deeper explanation for this different behavior at 
low and high magnetic fields. It is worth mentioning that the independent particle Hamiltonian can be exactly solved when only the Rashba or Dresselhaus SO terms are included. ${ }^{30}$ The merit of Eqs. (9)-(11) is that they are exact to the relevant $\lambda_{R, D}^{2}$ order when both SO couplings are simultaneously taken into account.

The excitation energy $\omega\left(S_{-}\right)$is the independent particle (KS) value for the spin splitting and violates Larmor's theorem even if the SO coupling is neglected. On the contrary, when the residual interaction is properly taken into account, the theorem Eq. (4) is recovered. In the following, we will concentrate on the large $B$ limit. It is then possible to derive the spin wave dispersion relation, including spin-orbit effects, by solving the equation

$$
1-2 F_{x c} \chi_{t}^{K S}(q, \omega)=0
$$

that gives the poles of the transverse response function Eq. (7). To do so, we write the transverse spin response $a^{27}$

$$
\mathcal{A} \chi_{t}^{K S}(q, \omega)=\frac{\left|\left\langle\omega\left(S_{-}\right)\left|\frac{1}{2} \sum_{j=1}^{N} e^{i \mathbf{q} \cdot \mathbf{r}} \sigma_{-}^{j}\right| 0\right\rangle\right|^{2}}{\omega-\omega\left(S_{-}\right)},
$$

where $\left|\omega\left(S_{-}\right)\right\rangle \equiv O^{+}\left[\omega\left(S_{-}\right)\right]|0\rangle$, and the corresponding energy is given by Eq. (10). The calculation of the matrix element in Eq. (13) must be done with care since $|0\rangle$ and $\left|\omega\left(S_{-}\right)\right\rangle$are not eigenstates of $S_{z}$ because of the spin-orbit coupling. Neglecting terms in $\lambda_{R, D}^{2} / \omega_{c}$ or smaller, one gets ${ }^{31}$

$$
\chi_{t}^{K S}(q, \omega)=\xi \frac{|F(q)|^{2}}{\omega-\omega\left(S_{-}\right)},
$$

where

$$
F(q)=\frac{1}{N}\left\langle 0\left|\sum_{j=1}^{N} e^{i \mathbf{q} \cdot \mathbf{r}_{j}}\right| 0\right\rangle
$$

is the gs elastic form factor. Note that $F(0)=1$ and that $F(q)$ goes to zero when $q \rightarrow \infty$. From Eq. (12) one finally obtains

$$
\begin{aligned}
\omega= & \left|g^{*} \mu_{B} B\right|+2 \lambda_{R}^{2} \frac{\omega_{c}}{\omega_{c}+\widetilde{\omega}_{L}}-2 \lambda_{D}^{2} \frac{\omega_{c}}{\omega_{c}-\widetilde{\omega}_{L}} \\
& -2 W_{x c}\left[1-|F(q)|^{2}\right] .
\end{aligned}
$$

This is the main result of our work, together with the lack of SO splitting we have found in the small $B$ limit. In the limit $q \rightarrow \infty$, Eq. (16) yields the independent particle spin splitting Eq. (10), which crucially depends on the actual value of $W_{x c}$ entering the definition of $\widetilde{\omega}_{L}$. In the $q=0$ limit, neglecting terms of order $\widetilde{\omega}_{L} / \omega_{c}$, Eq. (16) reduces to ${ }^{31}$

$$
\omega=\left|g^{*} \mu_{B} B\right|+2\left(\lambda_{R}^{2}-\lambda_{D}^{2}\right) .
$$

This expression shows that, neglecting the SO coupling, Larmor's theorem is fulfilled in the adiabatic TDLSCDA (it can be shown that the same holds in the adiabatic TDLSDA), and that at high magnetic fields, the SO interaction yields a $B$ independent contribution to the spin splitting. Taking, e.g., $m \lambda_{R}^{2} / \hbar^{2}=27 \mu \mathrm{eV}, m \lambda_{D}^{2} / \hbar^{2}=6 \mu \mathrm{eV}$, which have been recently used to reproduce the spin splitting in quantum dots ${ }^{14}$ and the splitting of the cyclotron resonance in quantum

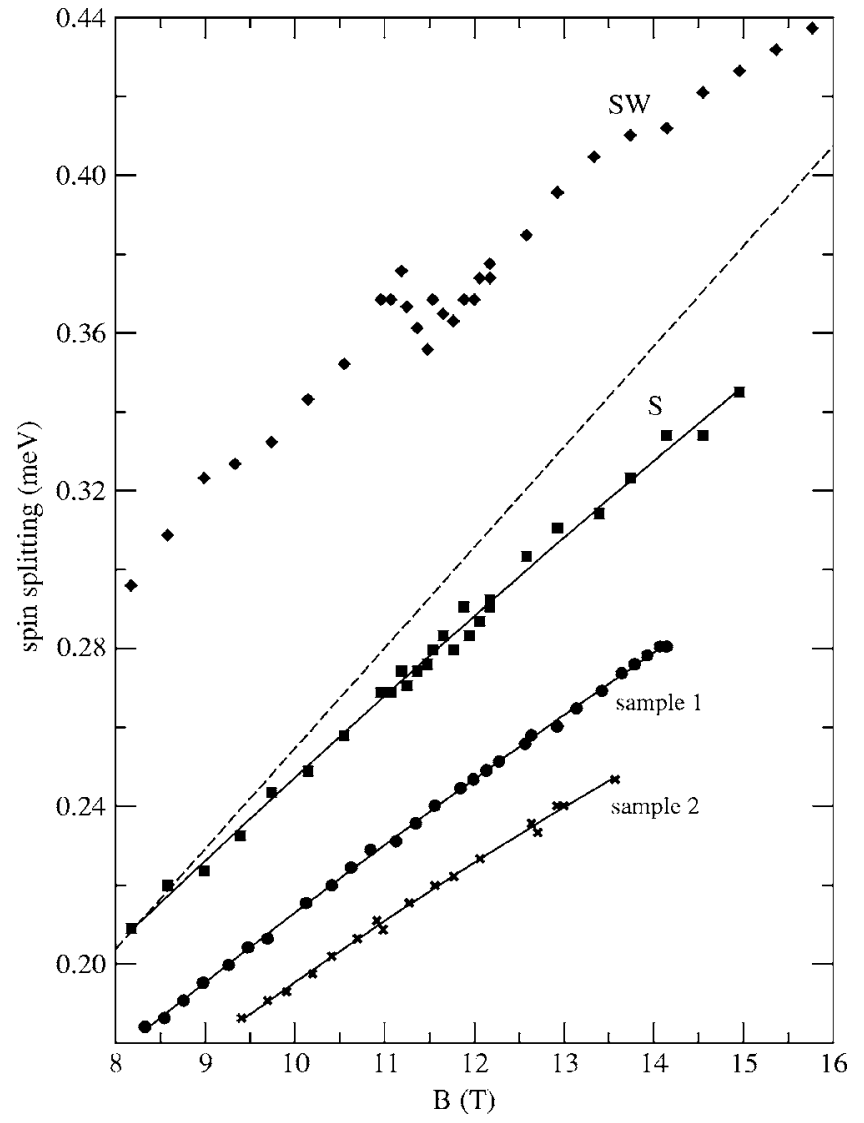

FIG. 1. Experimental spin splittings as a function of $B$. Squares and diamonds, from Ref. 21; dots (sample 1) and crosses (sample 2 ), from Ref. 24. The dashed line corresponds to $\mu_{B} g^{*} B$ with $\left|g^{*}\right|$ $=0.44$, and the solid lines are fits using the law Eq. (17).

wells, ${ }^{17}$ we get $2 m\left(\lambda_{R}^{2}-\lambda_{D}^{2}\right) / \hbar^{2} \sim 40 \mu \mathrm{eV}$. This is definitely a small amount, but it may have an influence on the fine analysis of some experimental results (note the vertical scale in Fig. 1).

\section{COMPARISON WITH EXPERIMENTS AND DISCUSSION}

Using inelastic light scattering, Davies et al. ${ }^{21}$ and Kang et al. $^{22}$ have measured charge and spin density excitation in two-dimensional (2D) electron systems confined in GaAs quantum wells at high $B$. In the following we only discuss the results of Ref. 21 because the information presented in Fig. 2 of this reference is especially well suited for the purpose of our work. These results are represented in Fig. 1. The data labeled $S$ correspond to wave vector allowed scattering from the $q=0$ Larmor mode; indeed, the maximum in plane $q$ allowed by the experimental geometry is small, $q_{\max }$ $=6 \times 10^{4} \mathrm{~cm}^{-1}$, so that the $S$ mode of energy $\epsilon_{S}$ should correspond to the spin splitting energy Eq. (17). The data labeled $S W$ is attributed to disorder-activated scattering, and would correspond to $q \neq 0$ excitations of energy $\epsilon_{S W} \cdot{ }^{21}$ The difference between $\epsilon_{S W}$ and $\epsilon_{S}$ is attributed to the exchange enhancement of $\epsilon_{S W}$ above the Zeeman energy. ${ }^{21}$ The dashed straight line represents the Larmor energy taking for $g^{*}$ the 
TABLE I. Parameters of the least-square linear fit to the cited experimental data.

\begin{tabular}{lccc}
\hline \hline & $\begin{array}{c}\hbar^{2} m\left(\lambda_{R}^{2}-\lambda_{D}^{2}\right) \\
(\mu \mathrm{eV})\end{array}$ & $g_{0}^{*}$ & $\begin{array}{c}r \\
\left(\mathrm{~T}^{-1}\right)\end{array}$ \\
\hline$\epsilon_{S}$ (Ref. 21) & 8.6 & 0.43 & $-7.3 \times 10^{-3}$ \\
Sample 1 (Ref. 24) & 7.7 & 0.38 & $-8.1 \times 10^{-3}$ \\
Sample 2 (Ref. 24) & -1.3 & 0.41 & $-1.3 \times 10^{-2}$ \\
\hline \hline
\end{tabular}

bulk value, $\left|g^{*}\right|=0.44$. This overestimates $\epsilon_{S}$, especially at high $B$.

Any sensible comparison with these results must take into account the $B$ dependence of $g^{*}$. This dependence has been clearly established in magnetoresistivity experiments, ${ }^{24}$ taking advantage of the fact that the electric-spin resonance affects the magnetoresistivity of the $2 \mathrm{D}$ electron gas, and this can be used to determine $g^{*}(B)$. These experiments probe the one-electron energy levels and are not influenced by manyelectron interactions, contrarily to magnetoquantum oscillations, which are strongly influenced by many-electron interactions.

The spin splitting obtained in Ref. 24 is represented in Fig. 1 as dots and crosses, which correspond to two different samples. In the lowest Landau level, which is the common physical situation for all data represented in Fig. 1, these authors have fitted $g^{*}(B)$ as $\left|g^{*}(B)\right|=g_{0}^{*}+r B / 2$. The value of the parameters $r$ and $g_{0}^{*}$ turns out to sensibly depend on the experimental sample, and the possibility of a SO shift at high $B$ values could not be considered there. Moreover, the $\lambda_{R, D}$ values are rather poorly known and dependent on, e.g., the thickness of the experimental sample.

We have thus renounced to use the laws $g^{*}(B)$ obtained in Ref. 24 in conjunction with Eq. (17), to establish a clear evidence of spin-orbit effects on the $\epsilon_{S}$ energy obtained from resonant inelastic light scattering experiments, and have satisfied ourselves with the more limited scope of using Eq. (17) as a three-parameter law to fit $\epsilon_{S}$ as well as the spin splittings of Ref. 24, with the aim of seeing whether a reasonable value for these parameters can be extracted.

The solid straight lines in Fig. 1 represent the result of such linear fits, whose parameters are collected in Table I. In the case of inelastic light scattering, the neglect of the SO term in Eq. (17) yields an unrealistic $g_{0}^{*}=0.49$, as this value should be smaller than that of bulk GaAs due to the penetration of the electron wave functions into the $\mathrm{Al}_{x} \mathrm{Ga}_{1-x} \mathrm{As}$ bar- riers. The dispersion of the electron-spin resonance datapoints ${ }^{24}$ seems to be smaller, and the analysis of the high $B$ data might be used to ascertain which $\mathrm{SO}$ mechanism is dominating in a given sample. This could be an alternative or complementary method to the recently proposed ${ }^{14}$ using the anisotropy of the spin splitting in single-electron resonant tunneling spectroscopy in lateral quantum dots submitted to perpendicular or parallel magnetic fields. The analysis of samples 1 and 2 would indicate that in the former, the Dresselhaus SO is the dominating mechanism, whereas in the latter it is the Bychkov-Rashba one. We want to stress that we have extracted the experimental data from a careful digitalization of the original figures. Due to the smallness of the effects we are discussing, we cannot discard that this procedure may have had some effect on the value of the parameters determined from the fit, and our analysis should be considered as qualitative to some extent. However, we find it encouraging that the parameters obtained from the fit are meaningful, and within the range of values found in other works. ${ }^{14,17}$

We finally discuss briefly the $q \neq 0 S W$ mode. From Eqs. (16) and (17), we have that $\epsilon_{S W}-\epsilon_{S}=-2 W_{x c}\left[1-|F(q)|^{2}\right]$. At high $q$, this difference is sensibly determined by $W_{x c}$. In our calculation, as well as in time-dependent Hartree-Fock ${ }^{29}$ and exact diagonalization ${ }^{32}$ calculations, we have found values of $W_{x c}$ of the order of $-2 \mathrm{meV}$. Hence, $-2 W_{x c}$ is about a factor 40 larger than the measured $\epsilon_{S W}-\epsilon_{S}$, which has been obtained in the $q \rightarrow 0$ limit where short range correlations are very important in determining the actual value of $1-|F(q)|^{2}$. This can be seen by assuming for $F(q)$ the independent particle value. Using a Slater determinant made of Fock-Darwin single particle wave functions to describe the gs of the system at $B \neq 0$, one finds $F(q \ell)=e^{-q^{2} \ell^{2} / 4}$, where $\ell$ is the magnetic length $\ell=(\hbar c / e B)^{1 / 2}$. In the small $q$ limit, $\epsilon_{S W}-\epsilon_{S} \simeq$ $-W_{x c} q^{2} \ell^{2}$. For $B=10 \mathrm{~T}, \quad q_{\max } \ell \simeq 0.05$, and $\epsilon_{S W}-\epsilon_{S}$ $\sim 0.01 \mathrm{meV}$, which is about one tenth of the experimental result as shown in Fig. 1. Light scattering experiments at small $q$ and high $B$ are thus very sensitive to correlation effects in the elastic form factor, which is the key quantity to reproduce the experimental findings.

\section{ACKNOWLEDGMENTS}

This work has been performed under Grant No. FIS200501414 from DGI (Spain) and Grant No. 2005SGR00343 from Generalitat de Catalunya. E.L. has been suported by DGU (Spain), Grant No. SAB2004-0091.
*Permanent address: Dipartimento di Fisica, Università di Trento, and INFN, 38050 Povo, Trento, Italy.

${ }^{1}$ C-M. Hu, J. Nitta, T. Akazaki, H. Takayanaga, J. Osaka, P. Pfeffer, and W. Zawadzki, Phys. Rev. B 60, 7736 (1999).

${ }^{2}$ D. Richards and B. Jusserand, Phys. Rev. B 59, R2506 (1999).

${ }^{3}$ E. de Andrada e Silva, Phys. Rev. B 60, 8859 (1999).

${ }^{4}$ A. Voskoboynikov, S. S. Liu, C. P. Lee, and O. Tretyak, J. Appl. Phys. 87, 1 (2000).
${ }^{5}$ A. G. Mal'shukov and K. A. Chao, Phys. Rev. B 61, R2413 (2000).

${ }^{6}$ P. N. Racec, T. Stoica, C. Popescu, M. Lepsa, and T. G. van de Roer, Phys. Rev. B 56, 3595 (1997).

${ }^{7}$ O. Voskoboynikov, C. P. Lee, and O. Tretyak, Phys. Rev. B 63, 165306 (2001).

${ }^{8}$ J. A. Folk, S. R. Patel, K. M. Birnbaum, C. M. Marcus, C. I. Duruöz, and J. S. Harris, Jr., Phys. Rev. Lett. 86, 2102 (2001). 
${ }^{9}$ B. I. Halperin, A. Stern, Y. Oreg, J. N. H. J. Cremers, J. A. Folk, and C. M. Marcus, Phys. Rev. Lett. 86, 2106 (2001).

${ }^{10}$ I. L. Aleiner and V. I. Fal'ko, Phys. Rev. Lett. 87, 256801 (2001).

${ }^{11}$ M. Valín-Rodríguez, A. Puente, Ll. Serra, and E. Lipparini, Phys. Rev. B 66, 165302 (2002).

${ }^{12}$ M. Valín-Rodríguez, A. Puente, and Ll. Serra, Phys. Rev. B 66, 045317 (2002).

${ }^{13}$ M. Valín-Rodríguez, A. Puente, Ll. Serra, and E. Lipparini, Phys. Rev. B 66, 235322 (2002).

${ }^{14}$ J. Könemann, R. J. Haug, D. K. Maude, V. I. Fal'ko, and B. L. Altshuler, Phys. Rev. Lett. 94, 226404 (2005).

${ }^{15}$ S. Datta and B. Das, Appl. Phys. Lett. 56, 665 (1990).

${ }^{16}$ B. E. Kane, Nature (London) 393, 133 (1998).

${ }^{17}$ P. Tonello and E. Lipparini, Phys. Rev. B 70, 081201(R) (2004).

${ }^{18}$ G. Dresselhaus, Phys. Rev. 100, 580 (1955).

${ }^{19}$ Yu. A. Bychkov and E. I. Rashba, J. Phys. C 17, 6039 (1984).

${ }^{20}$ F. G. Pikus and G. E. Pikus, Phys. Rev. B 51, 16928 (1995).

${ }^{21}$ H. D. M. Davies, J. C. Harris, J. F. Ryan, and A. J. Turberfield, Phys. Rev. Lett. 78, 4095 (1997).
${ }^{22}$ M. Kang, A. Pinczuk, B. S. Dennis, M. A. Eriksson, L. N. Pfeiffer, and K. W. West, Phys. Rev. Lett. 84, 546 (2000).

${ }^{23}$ D. Stein, K. v. Klitzing, and G. Weimann, Phys. Rev. Lett. 51, 130 (1982)

${ }^{24}$ M. Dobers, K. v. Klitzing, and G. Weimann, Phys. Rev. B 38, 5453 (1988).

${ }^{25}$ M. Pi, F. Ancilotto, E. Lipparini, and R. Mayol, Physica E (Amsterdam) 24, 297 (2004).

${ }^{26}$ M. Ferconi and G. Vignale, Phys. Rev. B 50, 14722 (1994).

${ }^{27}$ E. Lipparini, Modern Many Particle Physics-Atomic Gases, Quantum Dots and Quantum Fluids (World Scientific, Singapore, 2003).

${ }^{28}$ C. Kallin and B. I. Halperin, Phys. Rev. B 30, 5655 (1984).

${ }^{29}$ A. H. MacDonald, J. Phys. C 18, 1003 (1985).

${ }^{30}$ J. Schliemann, J. C. Egues, and D. Loss, Phys. Rev. B 67, 085302 (2003), and references therein.

${ }^{31} \mathrm{We}$ have checked that the results presented in Sec. III remain unchanged if these terms are kept in the calculation.

${ }^{32}$ E. H. Rezayi, Phys. Rev. B 36, 5454 (1987). 